Trauma Berufskrankh 2016 · [Suppl 2]:

18:S189-S192

DOI 10.1007/s10039-015-0062-0

Online publiziert: 2. Juli 2015

๑) Springer-Verlag Berlin Heidelberg 2015

CrossMark

Die Definition einer Pseudarthrose wurde in der Literatur vielfach beschrieben. Im deutschsprachigen Raum wird die Pseudarthrose als Ausbleiben einer knöchernen Überbrückung 6 Monate oder länger nach einer Fraktur definiert [10]. Zur Ätiologie der Pseudarthrose können

2 Faktoren angeführt werden:

1. Mechanische Faktoren wie die Interposition von Weichteilen in den Frakturspalt, eine starke Dislokation oder Distraktion der Fraktur im Bereich des Humerusschafts auch nach Osteosynthese und eine zu geringe Kompression auf den Frakturspalt

2. Mangelhafte Ruhigstellung bzw. zu frühe Mobilisierung nach durchgeführter Osteosynthese.

Als begünstigende Faktoren werden eine ungenügende Blutversorgung, das Auftreten einer Infektion, ein Gewebeverlust bei offenen Frakturen sowie systemische Erkrankungen wie Diabetes mellitus oder auch eine periphere arterielle Verschlusskrankheit genannt. Um die Häufigkeit einer Pseudarthrose angeben zu können,

\title{
L. Schütz
}

Klinik für Orthopädie, Unfall- und Handchirurgie, Klinikum Chemnitz, Chemnitz

\section{Pseudarthrosen am Humerusschaft}

ist zunächst die Inzidenz von Humerusschaftfrakturen zu beziffern, infolge derer sich dann eine Pseudarthrose bei entsprechender Therapie ausbilden kann.

\section{Häufigkeit und Ätiologie}

Bezüglich der Epidemiologie der Humerusschaftfraktur sind in der Literatur vereinzelt Studien angeführt. So geben Ekholm et al. [11] die Häufigkeit mit 14,5 Fällen pro 100.000 Einwohner pro Jahr an. Hier räumen die Autoren allerdings ein, dass ab dem 5. Lebensjahrzehnt ein Anstieg von 60 Fällen pro 100.000 Einwohner bis zum 9. Lebensjahrzehnt zu beobachten ist. Eine andere Studie beziffert die Häufigkeit von Humerusschaftfrakturen mit 1,9\% [3]. Um der Frage nachzugehen, wie häufig Pseudarthrosen auftreten, lässt sich dies nur durch die Studien über die Behandlung der Humerusschaftfrakturen feststellen.

Als weiterer Gesichtspunkt kann hier auch die Frage aufgeworfen werden, ob eine Häufung von Pseudarthrosen bei be- stimmten Osteosyntheseformen zu beobachten ist.

\section{Konservative Behandlung}

Eine Möglichkeit der Behandlung der Humerusschaftfraktur ist die konservative Versorgung, die durch eine Gips- oder Brace-Behandlung erfolgen kann. Bereits im Lehrbuch von Böhler [4] sind einige Beispiele einer konservativen Behandlung der Humerusschaftfraktur beschrieben. Die in neuerer Zeit verwendete Brace-Behandlung ist eine von Sarmiento [20] inaugurierte Methode, die allerdings sehr schnell an ihre Grenzen stößt. Als Ausschlusskriterien zu nennen sind hier Schaftquerfrakturen, kurze Schrägfrakturen mit einem erhöhten Pseudarthroserisiko, Adipositas, ein fehlender Muskelmantel, der für die optimale Schienung der Fraktur unerlässlich ist, komplizierte und metaphysär gelegene Frakturen sowie eine fehlende Compliance des Patienten. All diese Faktoren können neben einer Frakturdislokation auch die unter der Gips- bzw. Brace-Behandlung beobach-
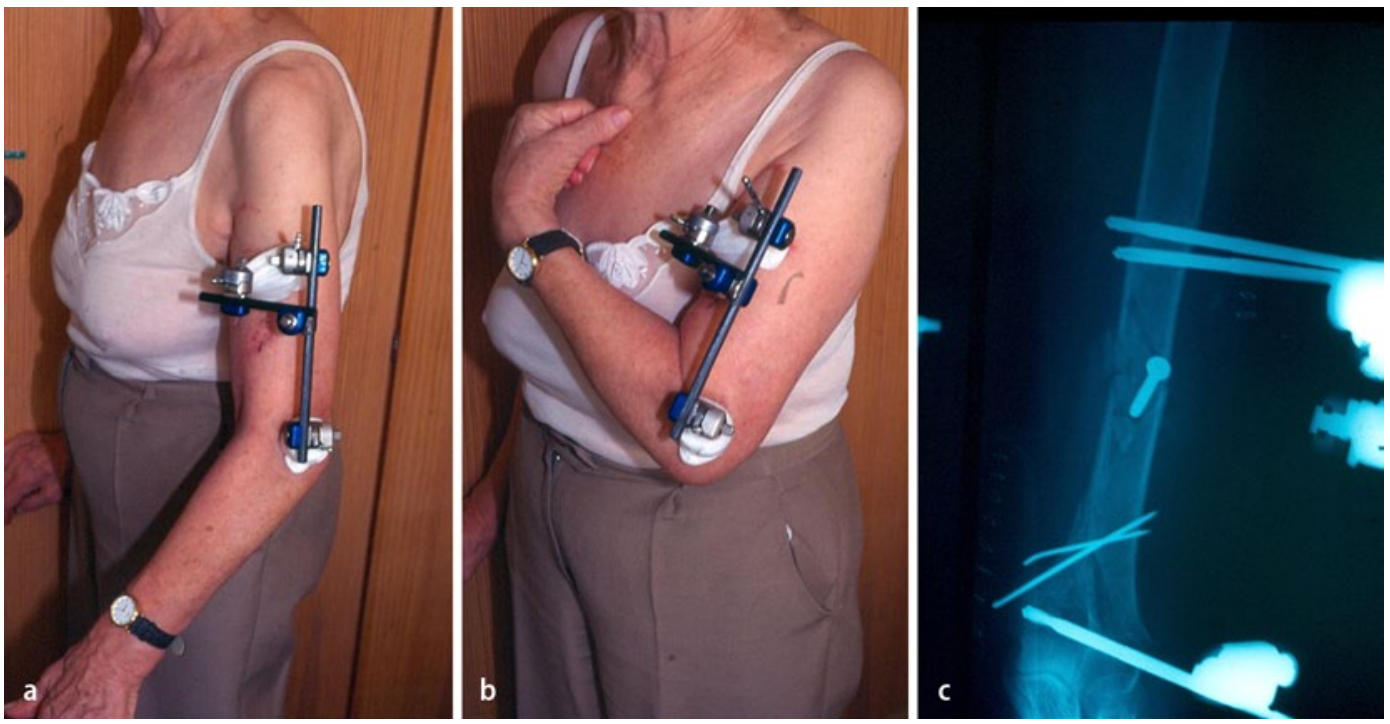

Abb. $1<$ a-c Versorgungsbeispiel eines unilateral angelegten Fixateur externe in Kombination mit einer minimal-invasiven Osteosynthese 

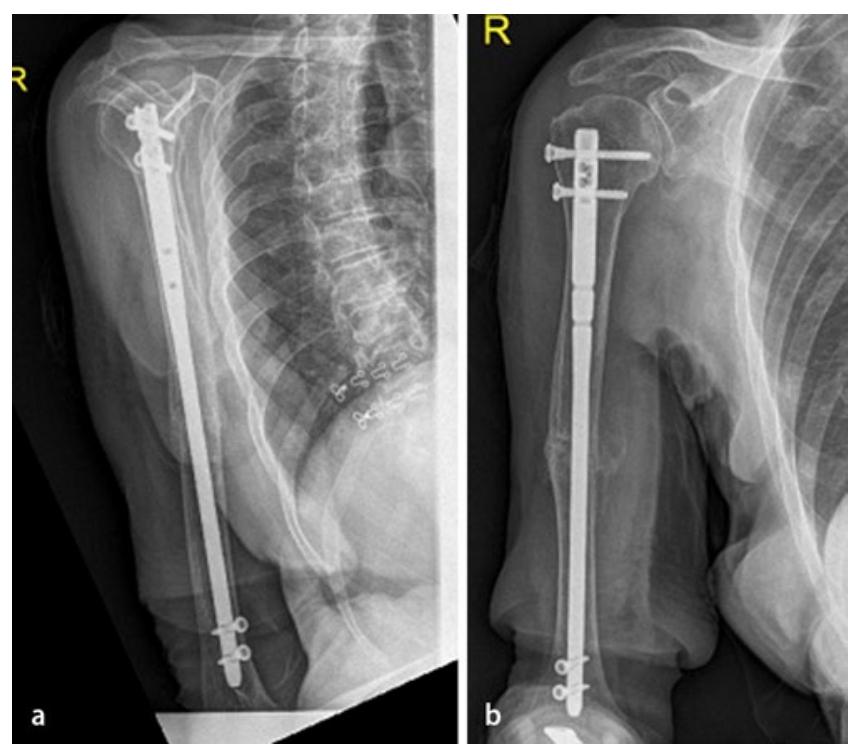

Abb. $2<$ a,b Versorgungsbeispiel einer Humerusfraktur stabilisiert mit einem antegrad eingebrachten Verriegelungsnagel
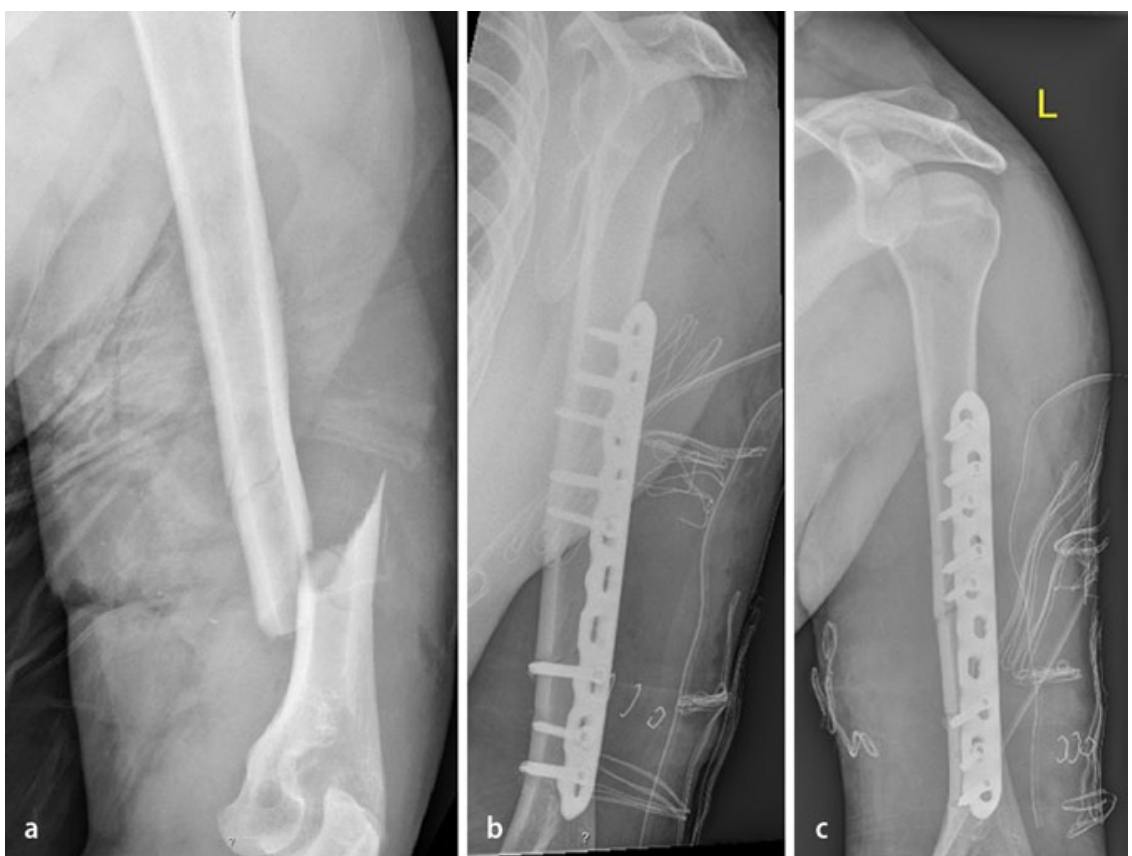

Abb. $3 \Delta$ a-c Versorgungsbeispiel einer Plattenosteosynthese

teten Hautprobleme sowie eine schmerzbedingte Pseudoparalyse bedingen. In der Literatur ist beim Vergleich zwischen den konservativen und den operativen Verfahren eine Studie anzuführen, in deren Patientengut eine höhere Rate an Pseudarthrosen bei konservativer Behandlung beobachtet wurde [9].

\section{Operative Behandlung}

Als mögliche operative Verfahren kommt laut Literatur neben seltenen Verfahren die Therapie mit dem Fixateur externe, mit intramedullären Implantaten bzw. einer Plattenosteosynthese als offenes Verfahren infrage. Die Behandlung mit dem Fixateur externe [1] ist eine durchaus erfolgreiche Methode, die v. a. bei drittgradig offenen Frakturen bzw. bei erheblichem Infektionsrisiko eingesetzt werden kann (• Abb. 1). Allerdings erscheint die Fixateurbehandlung für die Patienten mitunter belastend, da hier regelmäßige Konsultationen auch in einem engeren zeitlichen Abstand erforderlich sind und zudem die Compliance des Patienten, die tägliche Pflege des Fixateurs durchzufüh- ren, vorhanden sein muss. Ein etabliertes Verfahren ist die Nagelosteosynthese (• Abb. 2), wobei hier die Nagelung sowohl antegrad als auch retrograd erfolgen kann. Als Nachteile der Nagelosteosynthese werden in der Literatur die Affektion im Bereich des Schultergürtels bei antegradem Vorgehen bzw. auch Affektionen der Nerven im Schultergürtelbereich durch die einzubringenden Verriegelungsschrauben beschrieben [13]. Die Plattenosteosynthese ist ein standardisiertes Verfahren (• Abb. 3), wobei hier die ausgedehnte Exposition der Weichteile mit den dadurch verbundenen möglichen Komplikationen zu beachten ist. In einer Metaanalyse sind Studien aufgeführt, bei denen Plattenosteosynthese und Nagelosteosynthese gleichwertige Ergebnisse zeigen, dies gilt auch für eine Level-II-Studie [15]. Beschrieben ist allerdings ebenfalls, dass Nagelosteosynthesen v. a. beim antegradem Vorgehen eine höhere Rate an Schulteraffektionen aufweisen.

In mehreren Studien wird die Rate der Pseudarthrosen bei Osteosynthesen mit einer unterschiedlichen Anzahl angegeben $[8,17,19,20]$. Diese schwankt zwischen $5,2 \%$ bzw. $6,5 \%$ bis zu $10 \%$. In einer neueren Studie [6] wird von Raten bis zu 12,5\% berichtet. Die von Bolano [5] beschriebene Pseudarthroserate von fast $28 \%$ bei Nagelosteosynthesen ist sicherlich auch auf die im Jahre 1995 fehlende Erfahrung in der Operationstechnik zurückzuführen.

\section{Diagnostik}

Pseudarthrosen führen häufig zu dauerhaften Funktionseinschränkungen und anhaltenden Schmerzen im Bereich des Humerusschafts. Weitere Merkmale sind eine abnorme Beweglichkeit und ein im Röntgenbild sichtbarer Pseudarthrosenspalt mit reaktiver Sklerosierung in der Umgebung des Röntgenbilds.

Zur Diagnostik der Pseudarthrose ist das konventionelle Röntgen in 2 Ebenen $\mathrm{zu}$ fordern, wobei hier mitunter insbesondere bei Einliegen von Osteosynthesematerial erhebliche Überlagerungen bestehen können (• Abb. 4). In diesem Fall ist eine Computertomographie als Schnittbildtechnik, ggf. eine Magnetresonanztomographie oder aber bei Verdacht 
auf eine Infektpseudarthrose auch eine Knochenszintigraphie zur präoperativen Diagnosestellung sinnvoll. Bei Pseudarthrosen werden 2 Formen unterschieden, die in der Bildgebung sichtbar werden: erstens die atrophe, nichtreaktive Pseudarthrose mit vollständigem Fehlen von knöchernen Reaktionen bzw. die avaskuläre/avitale Pseudarthrose mit oder ohne Knochenverlust sowie zweitens die reaktive hypertrophe Pseudarthrose mit vermehrter Sklerose der Knochenenden und überschießender Knochenneubildung.

Die Pseudarthrosenform bestimmt auch die weiterführende Therapie. So ist bei der vitalen Pseudarthrose das Pseudarthrosengewebe zu belassen, es sollte eine achsengerechte Stabilisierung und vorzugsweise eine Plattenosteosynthese erfolgen. Bei einer avitalen Pseudarthrose ist die Transplantation von autogenem Knochenmaterial bzw. Knochenersatzmaterial oder ggf. auch die Applikation von Wachstumsfaktoren unerlässlich.

\section{Verfahrenswahl}

Zur Verfahrenswahl bei der Pseudarthrose ist zunächst der Fixateur externe zu nennen. Mit dieser Methode zeigt sich in der Literatur, zuletzt von Atalar et al. [2] beschrieben, bei korrekter Applikation stets eine gute Heilung. Allerdings sind bei Verwendung des Fixateur externe Infektionen, Fehlstellungen bzw. Refrakturen möglich. Auch weisen die Autoren auf mögliche Gelenkeinsteifungen der benachbarten Gelenke hin. Hinsichtlich der Entscheidung zwischen Nagel- und Plattenosteosynthese findet sich in der Literatur überwiegend eine Favorisierung der Plattenosteosynthese, wobei hier insbesondere darauf hingewiesen wird, dass die besten Ergebnisse durch eine Plattenosteosynthese und gleichzeitige Durchführung einer autologen Spongiosaplastik erreicht werden [7]. Insbesondere wird in vielen Studien die korrekte Anwendung der Plattenosteosynthese durch exzentrisches Bohren der Bohrlöcher, aber auch durch die Verwendung eines Plattenspanngeräts erwähnt. Dies ist zur Erreichung einer hohen Kompression im interfragmentären Pseudarthrosenspalt erforderlich [14, 16, 18]. Bezüglich der Verwendung von autologer Spongio- sa bzw. demineralisierter Knochenmatrix finden sich in einer Level-III-Studie keine wesentlichen Unterschiede zwischen beiden Verfahren [12].

$\mathrm{Zu}$ beachten ist die Pseudarthrosenlokalisation sowie -aktivität. Ebenso stellen in der präoperativen Planung die Knochenqualität sowie die vorhandenen Weichteilverhältnisse wesentliche Faktoren dar.

Ein weiteres, seltener angewendetes Verfahren ist der Segmenttransport, der allerdings für den Patienten auch aufgrund der Langwierigkeit der Behandlung nur wenig attraktiv ist. Er ist v. a. bei ausgedehnten Infektpseudarthrosen eine Behandlungsoption. Die Elektrostimulation bzw. extrakorporale Stoßwellentherapie wird nach anfänglicher Begeisterung mittlerweile nicht mehr breit angewendet. Bezüglich der bei malignen Tumoren auftretenden Pseudarthrosen ist das Verfahren in Abhängigkeit von der klinischen Gesamtsituation des Patienten zu wählen. Dabei sollte unter Berücksichtigung der begrenzten Lebenserwartung des Patienten und der lokalen Tumorausbreitung eine höchstmögliche Lebensqualität für den Patienten gewährleistet werden.

Grundsätzlich gilt: Um Pseudarthrosen zu vermeiden, sollte präventiv eine korrekte Reposition bei der Primäroperation erfolgen [21].

\section{Korrespondenzadresse}

\section{Dr. L. Schütz}

Klinik für Orthopädie, Unfall- und Handchirurgie Klinikum Chemnitz, Bürgerstr. 2

09113 Chemnitz

I.schuetz@skc.de

\section{Einhaltung ethischer Richtlinien}

Interessenkonflikt. L. Schütz gibt an, dass kein Interessenkonflikt besteht.

Dieser Beitrag beinhaltet keine Studien an Menschen oder Tieren.

The supplement containing this article is not sponsored by industry.
Trauma Berufskrankh 2016 · [Suppl 2]:

18:S189-S192

DOI 10.1007/s10039-015-0062-0

c) Springer-Verlag Berlin Heidelberg 2015

\section{Schütz}

\section{Pseudarthrosen am Humerusschaft}

\section{Zusammenfassung}

Hintergrund. Die Behandlung der Pseudarthrosen orientiert sich an deren Ursache. Dabei sind immer individuelle Faktoren zu berücksichtigen, insbesondere auch lokale Faktoren wie Knochendichte, Weichteilverhältnisse bzw. Vorerkrankungen.

Therapie. Bei der Verfahrenswahl scheint sich die Plattenosteosynthese in Kombination mit einer autologen Spongiosaplastik auch in der Literatur als etabliertes Verfahren darzustellen. Bezüglich der Applikation der Platte, ob ventral oder dorsal, finden sich noch keine ausgedehnten Studien. Grundsätzlich ist zur Prävention bei der primären Osteosynthese einer Humerusschaftfraktur auf ein korrektes Vorgehen zu achten, insbesondere auch auf eine ausreichende Kompression im Frakturspalt.

\section{Schlüsselwörter}

Pseudarthrose $\cdot$ Humerusfraktur .

Frakturfixation - Knochenplatten .

Osteosynthese

\section{Pseudarthrosis of the humeral shaft}

\section{Abstract}

Background. The treatment of pseudarthrosis depends on the cause. Individual and local factors, such as bone density, the soft tissue situation and previous illnesses have to be considered.

Therapy. Humeral plating combined with an autologous spongiosa graft seems to be a well-established procedure even in the current literature. No extensive studies referring to plate fixation (ventral or dorsal) can currently be found. A correct approach for primary osteosynthesis of humeral shaft fractures is necessary for prevention of humeral pseudarthrosis especially in terms of sufficient compression of the fracture line.

Keywords

Pseudarthrosis · Humeral fracture · Fracture fixation - Bone plates - Osteosynthesis 

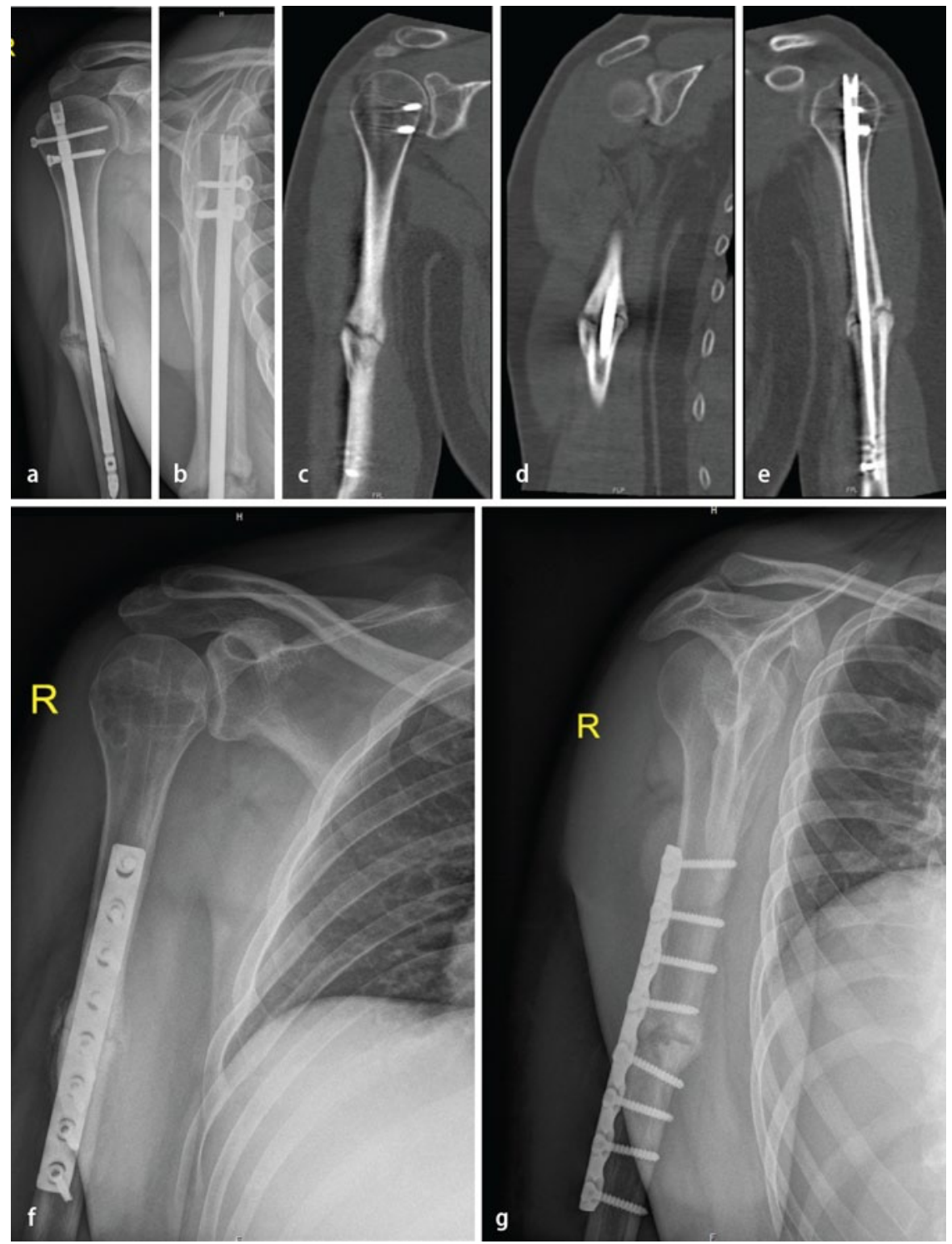

Abb. $4 \Delta$ Versorgungsbeispiel einer Pseudarthrose nach Verriegelungsnagelung. a,b Konventionelles Röntgenbild lässt eine hypertrophe Pseudarthrose 9 Monate nach Primärosteosynthese vermuten. c-e Computertomogramm präoperativ beweist die Diskontinuität. f,g Konventionelle Röntgenbilder postoperativ

\section{Literatur}

1. Asche G, Roth W, Schroeder L (2002) Fixateur externe. Einhorn, Schwäbisch Gmünd

2. Atalar AC, Kocaoglu M, Demirhan M, Bilsel K, Eralp $L$ (2008) Comparison of three different treatment modalities in the management of humeral shaft nonunions (plates, unilateral, and circular external fixators). J Orthop Trauma 22(4):248-257

3. Bercik MJ, Tjoumakaris FP, Pepe M, Tucker B, Axel$\operatorname{rad}$ A, Ong A, Austin L (2013) Humerus fractures at a regional trauma center: an epidemiologic study. Orthopedics 36(7):e891-e897

4. Böhler L (1996) Technik der Knochenbruchbehandlung. Maudrich, Wien
5. Bolano LE, laquinto JA, Vasicek V (1995) Operative treatment of humerus shaft fractures: a prospective randomized study comparing intramedullary nailing with dynamic compression plating. American Academy of Orthopaedic Surgeons Annual Meeting; 16-21 Feb 1995, Orlando

6. Changulani M, Jain UK (2008) Comparison of humerus intramedullary nail and dynamic compression plate for the management of diaphyseal fractures of humerus. A randomised prospective study. J Bone Joint Surg Br 90:349-350 domme G, Guinand C, Fontaine R (2005) Surgery for pseudarthrosis of humeral shaft fractures: a retrospective series of 21 cases. Chir Main 24(2):8491

8. Chapman JR, Henley MB, Agel J, Benca PJ (2000) Randomized prospective study of humeral shaft fracture fixation: intramedullary nails versus plates. J Orthop Trauma 14(3):162-166
7. Chantelot $C$, Ferry S, Lahoude-Chantelot S, Pro-
9. Denard A, Richards JE, Obremskey WT, Tucker MC, Floyd M, Herzog GA (2010) Outcome of nonoperative vs operative treatment of humeral shaft fractures: a retrospective study of 213 patients. Orthopedics $33(8)$

10. Döhler RJ, Pseudarthrose (Hrsg) (2003) Lexikon Orthopädische Chirurgie. Springer, Berlin Heidelberg New York, S 176-179

11. Ekholm R, Adami J, Tidermark J, Hansson K, Törnkvist $H$, Ponzer $S$ (2006) Fractures of the shaft of the humerus, an epidemiological study of 401 fractures. J Bone Joint Surg Br 88-B:1469-1473

12. Hierholzer C, Sama D, Toro JB, Peterson M, Helfet DL (2006) Plate fixation of ununited humeral shaft fractures: effect of type of bone graft on healing. J Bone Joint Surg Am 88(7):1442-1447

13. Kurup H, Hossain M, Andrew JG (2011) Dynamic compression plating versus locked intramedullary nailing for humeral shaft fractures in adults. Cochrane Database Syst Rev 15(6):CD005959

14. Lin CL, Fang CK, Chiu FY, Chen CM, Chen TH (2009) Revision with dynamic compression plate and cancellous bone graft for aseptic nonunion after surgical treatment of humeral shaft fracture. J Trauma 67(6):1393-1396

15. Ouyang H, Xiong J, Xiang P, Cui Z, Chen L, Yu B (2013) Plate versus intramedullary nail fixation in the treatment of humeral shaft fractures: an updated meta-analysis. J Shoulder Elbow Surg 22(3):387-395

16. Padhye KP et al (2013) Plating, nailing, external fixation, and fibular strut grafting for non-union of humeral shaft fractures. J Orthop Surg (HongKong) 21(3):327-331

17. Paris $\mathrm{H}$, Tropiano $\mathrm{P}$, Clouet D'orval $\mathrm{B}$, Chaudet $\mathrm{H}$, Poitout DG (2000) Fractures of the shaft of the humerus: systematic plate fixation. Anatomical and functional results in 156 cases and a review of the literature. Rev Chir Orthop Reparatrice Appar Mot 86(4):346-359

18. Ring D, Perey BH, Jupiter JB (1999) The functional outcome of operative treatment of ununited fractures of the humeral diaphysis in older patients. J Bone Joint Surg Am 81(2):177-190

19. Rommens PM, Blum $P$, Janzing $H$, Langendorff $H S$ (1998) Retrograde nailing of humerus shaft fractures with the unreamed humerus nail. An international multicenter study. Unfallchirurg 101(5):342352

20. Sarmiento A, Latta LL (1999) Functional fracture bracing. J Am Acad Orthop Surg 7(1):66-75

21. Segonds JM, Alnot JY, Masmejean E (2003) Aseptic non-union of humeral shaft fractures treated by plating and bone grafting. Rev Chir Orthop Rep Appar Mot 89(2):107-114 